\title{
A Study to Assess the Behavioural and Psychological Symptoms in Patients with Dementia
}

\author{
Dr. Manjit Singh ${ }^{1}$, Dr. Manmeet Kaur ${ }^{2}$, Dr. P.D. Garg ${ }^{3}$, Dr. Neeru Bala ${ }^{* 4}$ \\ ${ }^{1}$ Associate Professor, Department of Psychiatry, GMC Amritsar \\ ${ }^{2}$ Third year Postgraduate Student, Department of Psychiatry, GMC Amritsar \\ ${ }^{3}$ Professor, Department of Psychiatry, GMC Amritsar \\ ${ }^{4}$ Associate Professor and Head, Department of Psychiatry, GMC Amritsar \\ Corresponding Author: Dr. Neeru Bala; manmeetsidhu782@gmail.com
}

Received 21 July 2019;

Accepted 12 August 2019;

Published 16 October 2019

\begin{abstract}
Introduction: With the aging population, the prevalence of dementia is rising. The aim of the study was to assess the frequency of Behavioural and Psychological symptoms (BPSD) in patients with dementia. Materials and Methods: A semi-structured proforma was used to gather sociodemographic details including age, gender, educational status, economic status, living conditions, family and personal history of any psychiatric illness. Mini Mental Status Examination was administered to record the level of cognitive impairment. Neuropsychiatric Inventory (NPI) was used to assess the presence of behavioral and psychological symptoms of dementia (BPSD) in patients with dementia. Findings: Out of 54 patients, $53.7 \%$ were females and $46.3 \%$ were males. $61.12 \%$ were above 65 years of age. Most of them $51.85 \%$ were illiterate. $66.67 \%$ belong to rural background. 59.25\% came from nuclear families. MMSE score distribution showed that $51.85 \%$ belong to mild dementia group. Mean MMSE score was 16.12+/-4.778 SD. On Neuropsychiatric Inventory (NPI), most common symptoms were apathy (70. 37\%), agitation (64.8\%), sleep disturbances $(59.25 \%)$ followed by other symptoms. Mean item score was 27.84+/-17.58 SD. Mean distress score was $17.72+/-$ 8.99 SD. Conclusion: BPSD are universally present in patients with dementia and cause significant caregiver distress.
\end{abstract}

Keywords: Dementia, Behaviour and Psychological Symptoms of Dementia, Mini Mental Status Examination, Neuropsychiatric Inventory.

\section{Background}

Dementia is a syndrome due to disease of the brain, usually of a chronic or progressive nature, in which there is disturbance of multiple higher cortical functions, including memory, thinking, orientation, comprehension, calculation, learning capacity, language and judgement. Consciousness is not clouded. Impairments of cognitive function are commonly accompanied, and occasionally preceded, by deterioration in emotional control, social behavior or motivation. ${ }^{[1]}$

The primary requirement for diagnosis according to ICD-10 is evidence of a decline in both memory and thinking which is sufficient to impair personal activities of daily living. The impairment of memory typically affects the registration, storage and retrieval of new information, but previously learned and familiar material may be lost, particularly in later stages. Dementia is more than dysmnesia: there is also impairment of thinking and of reasoning capacity and a reduction in flow of ideas. The processing of incoming information is impaired, in that the individual finds it increasingly difficult to attend to more than one stimulus at a time, such as taking part in a conversation with several persons, and to shift the focus of attention from one topic to another. If dementia is the sole diagnosis, evidence of clear consciousness is required. The above symptoms and impairments should have been evident for at least 6 months for a confident clinical diagnosis of dementia to be made. $^{[1]}$

Dementia impacts personal, family and societal life. It reduces life span, induces caregiver's strain at family level and utilizes health care facility, inflicting strain on national income. It is expected that burden of dementia will be increasing in developing countries due to increase in longevity and increasing prevalence of risk factors such as hypertension, stroke and lifestyle changes. ${ }^{[2]}$

Cognitive impairment is the hallmark feature of dementia; but it also expresses itself through behavioral and psychological symptoms which include delusion, apathy, depression, anxiety and a range of other maladaptive behaviors. Behavioral and psychological symptoms of dementia seem to be challenging for the caregivers. It may become a source of caregiver's burden as well as lead to secondary discomfort and distress for the person with dementia. ${ }^{[3]}$

The number of people living with dementia worldwide in 2015 was estimated at 47.47 million, reaching 75.63 million in 2030 and 135.46 million in $2050 .^{[4]}$ 
Through meta-analysis of the available evidence, we estimate over 9.9 million new cases of dementia each year worldwide, implying one new case every 3.2 seconds. These new estimates are almost $30 \%$ higher than the annual number of new cases estimated for 2010 in the $2012 \mathrm{WHO} / \mathrm{ADI}$ report (7.7 million new cases, one every 4.2 seconds).

The regional distribution of new dementia cases is 4.9 million (49\% of the total) in Asia, 2.5 million (25\%) in Europe, 1.7 million (18\%) in the Americas, and 0.8 million (8\%) in Africa. The incidence of dementia increases exponentially with increasing age. For all studies combined, the incidence of dementia doubles with every 6.3 years increase in age, from 3.9 per 1000 person-years at age 60-64 to 104.8 per 1000 person-years at age $90^{+} .^{[5]}$

During the natural course of dementia, a heterogeneous group of clinical phenomena are subjectively experienced by the patient and/or observable by an examiner (e.g. caregiver, physician) consisting in disturbed emotions, mood, perception, thought, motor activity, and altered personality traits. These "neuropsychiatric symptoms, "according to the terminology most used in the United States, or" behavioral and psychological symptoms of dementia" (BPSD), as designated by the International Psychogeriatric Association, are very common and associated with high levels of distress both in dementia sufferers and their caregivers, as well as with adverse outcomes and increased use of health care resources. ${ }^{[6]}$

BPSD include agitation, aberrant motor behavior, anxiety, elation, irritability, depression, apathy, disinhibition, delusions, hallucinations, and sleep or appetite changes. The pathogenesis of BPSD has not been clearly delineated but it is probably the result of a complex interplay of psychological, social, and biological factors. ${ }^{[7]}$

A study by Huang, Wang, Liao in 2017 revealed that severity of BPSD was associated with different stages in dementia. The highest severity of global BPSD was seen in severe dementia $(\mathrm{CDR}=3)$, and decreased in severity when CDR equaled 4. Considering individual BPSD, delusion, agitation/aggression, apathy/indifference, aberrant motor behavior, nighttime behavior disturbances, and appetite and eating abnormalities were significantly different among stages of dementia. ${ }^{[8]}$

Romero and Garrido reviewed the importance of behavioural and pyschological symptoms in Alzheimer disease. This underscores the importance of BPSDs in dementia, and more specifically in $\mathrm{AD}$. A large majority of patients will experience these symptoms at some point during disease progression. BPSDs have a negative impact on patients' quality of life and well-being. Early diagnosis of these symptoms is essential to preventing adverse effects, providing early treatment (pharmacological or otherwise), and designing the most suitable care plan for these patients. $^{[9]}$

\section{Methods}

It was a hospital based cross sectional study in which 54 consecutive outdoor and indoor patients fulfilling the diagnostic criteria of dementia were taken from Department of Psychiatry, Government Medical College, Amritsar from a period of January 2018 to January 2019. Approval of institutional ethics committee was taken. After taking consent from patients or attendants, patients were enrolled in the study.

The socio-demographic profile and information about the illness were recorded and diagnosis was made according to ICD-10 criteria. Mini Mental Status Examination was administered to record the level of cognitive impairment. Based on MMSE score, dementia was categorized into mild (score of 18 to 24), moderate (score of 10 to 17) and severe (score of 9 or less) types. Neuropsychiatric Inventory (NPI) was used to assess the presence of behavioral and psychological symptoms of dementia (BPSD) in patients with dementia.

\section{Inclusion criteria}

1. Age 60 and above of both sexes

2. Those patients who presented to Psychiatry Department, both outpatient and inpatient, diagnosed as having dementia according to ICD-10 criteria.

\section{Exclusion criteria}

1. Patient/ family members not giving consent

2. Mentally retarded patient

3. Uncooperative patient

4. Individuals with severe medical/surgical condition/disorder.

\section{Instruments}

\section{Proforma for socio-demographic variables}

A semi-structured proforma was used to obtain information about the participants and gather their sociodemographic details including age, gender, educational status, economic status, living conditions, family and personal history of any psychiatric illness.

\section{Mini- Mental State Examination}

The MMSE is by some way the best known and most widely used measure of cognition in clinical practice worldwide. This scale can be easily administered by clinicians or researchers with minimal training, takes around $10 \mathrm{~min}$ and assesses cognitive function in the areas of orientation, memory, attention and calculation, language and visual construction. Patients score between 0 and 30 points, and cutoffs of $23 / 24$ have typically been used to show significant cognitive impairment. It is widely translated and used. ${ }^{[10]}$

\section{Neuropsychiatric Inventory}

The Neuropsychiatric Inventory (NPI) was developed to assess psychopathology in dementia patients. It evaluates 12 neuropsychiatric disturbances common in dementia: delusions, hallucinations, agitation, dysphoria, anxiety, apathy, irritability, euphoria, disinhibition, aberrant motor behavior, night-time behavior disturbances, and appetite and eating abnormalities. The severity and frequency of each neuropsychiatric symptom are rated on the basis of scripted questions administered to the patient's caregiver. The NPI also assesses the amount of caregiver distress engendered by each of the neuropsychiatric disorders. A total NPI score and a total caregiver distress score are calculated, in addition to the scores for the individual symptom domains. Content validity, concurrent validity, inter-rater reliability, and test-retest reliability of the NPI are established. ${ }^{[11]}$

\section{Statistical analysis}

The data was evaluated using Microsoft excel worksheet and percentage, proportion and standard deviation for each and every variable was calculated.

\section{Results}

Out of 54 patients, $53.7 \%$ were females and $46.3 \%$ were males (Figure 1.1). 61.12\% were above 65 years of age and $38.88 \%$ were under 65 years of age (Figure 1.2). Most of them $51.85 \%$ were illiterate (Figure1.3). 66.67\% belong to rural background (Figure 
1.4). $59.25 \%$ came from nuclear families. Socio-demographic variables of patients are described in Table-1.

MMSE score distribution showed that $51.85 \%$ belong to mild dementia group, $37.03 \%$ belong to moderate dementia group and $11.12 \%$ belong to severe dementia group (Figure 2). Mean MMSE score was $16.12+/-4.778$ SD (Table-2) which comes in moderate dementia group.

On Neuropsychiatric Interview (NPI) 70. 37\% showed apathy, $64.8 \%$ showed agitation, $59.25 \%$ showed sleep disturbances, $57.4 \%$ showed irritable behavior, $55.56 \%$ showed dysphoric symptoms, anxiety symptoms and appetite and eating abnormalities. Less common symptoms were aberrant motor behavior in $46.3 \%$, delusions $26 \%$, hallucinations and disinhibition in $13 \%$ and elation in $11.12 \%$. Mean item score was $27.84+/-17.58$ SD. Mean distress score was 17.72+/-8.99 SD.(Table-3). Mean distress score was 17.72+/-8.99 SD.

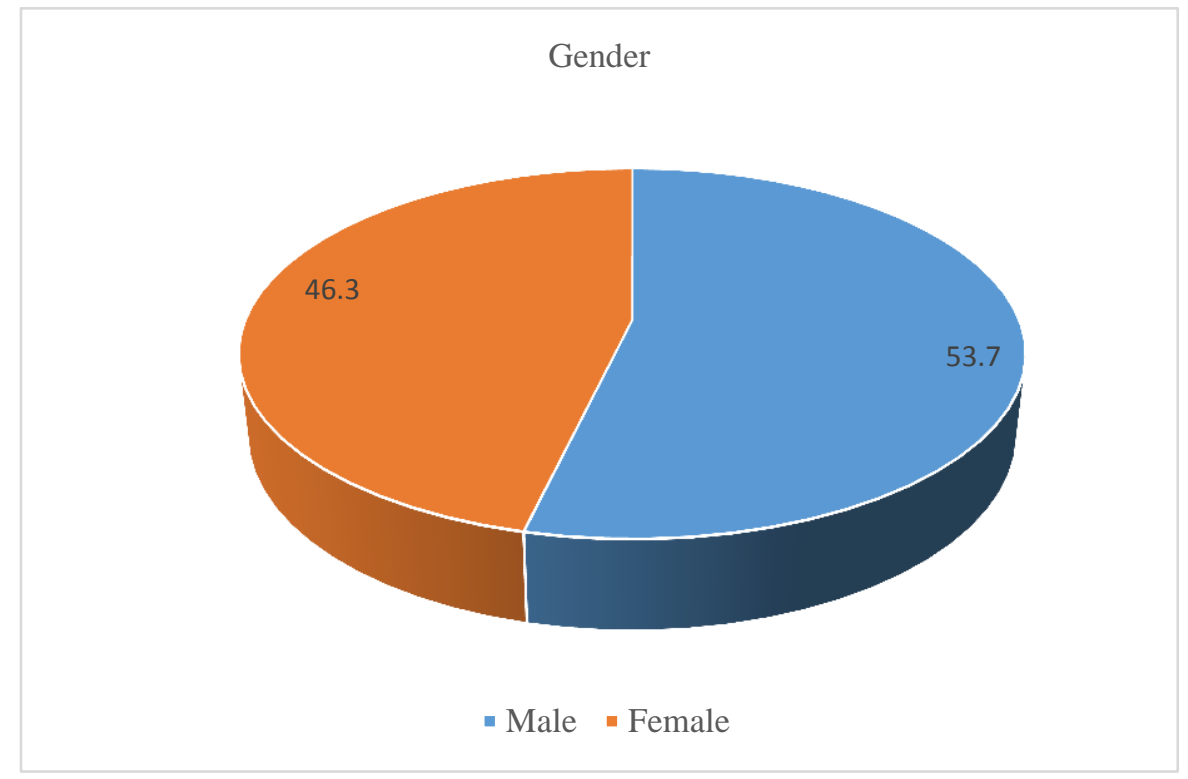

Figure: 1.1

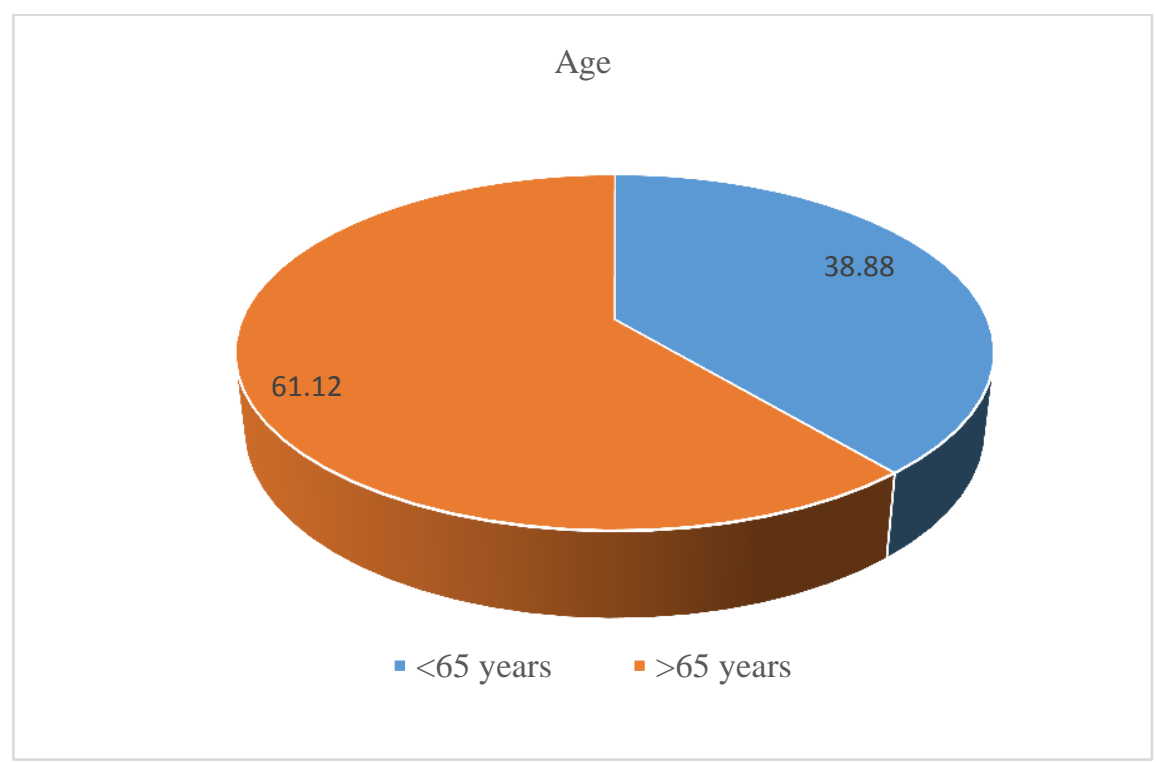

Figure: 1.2

Table 1: Distribution of sample on socio- demographic variables

\begin{tabular}{|l|l|l|l|}
\hline Category & Variables & Frequency & Percentage \\
\hline Gender & Female & 29 & 53.70 \\
& Male & 25 & 46.30 \\
& Total & 54 & 100 \\
\hline Age in years & $<65$ & 21 & 38.88 \\
& $>65$ & 33 & 61.12 \\
& total & 54 & 100 \\
\hline
\end{tabular}




\begin{tabular}{|l|l|l|l|}
\hline Educational status & Illiterate & 28 & 51.85 \\
& Primary & 15 & 27.78 \\
& Matric & 6 & 11.12 \\
& Higher sec & 3 & 5.56 \\
& Graduate & 2 & 3.7 \\
& Total & 54 & 100 \\
\hline Background & Rural & 36 & 66.67 \\
& Urban & 18 & 33.33 \\
& Total & 54 & 100 \\
\hline Family Type & Joint & 22 & 40.75 \\
& Nuclear & 32 & 59.25 \\
& Total & 54 & 100 \\
\hline Annual Family Income (Rs.) & Above 30,000/- & 44 & 81.48 \\
& Below 30,000/- & 10 & 18.52 \\
& Total & 54 & 100 \\
\hline Past H/O Psychiatric illness & No & 50 & 92.6 \\
& Yes & 4 & 7.4 \\
& Total & 54 & 100 \\
\hline Family H/O Psychiatric & No & 50 & 92.6 \\
illness & Yes & 4 & 7.4 \\
& Total & 54 & 100 \\
\hline
\end{tabular}

Education status

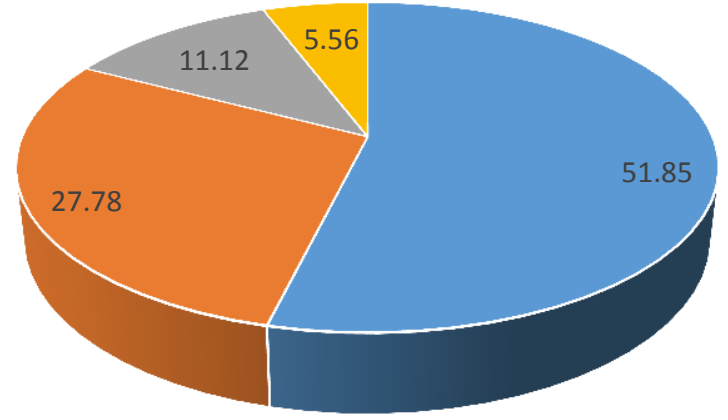

- Illiterate - Primary - Matric $=$ Higher Sec - Graduate

Figure: 1.3

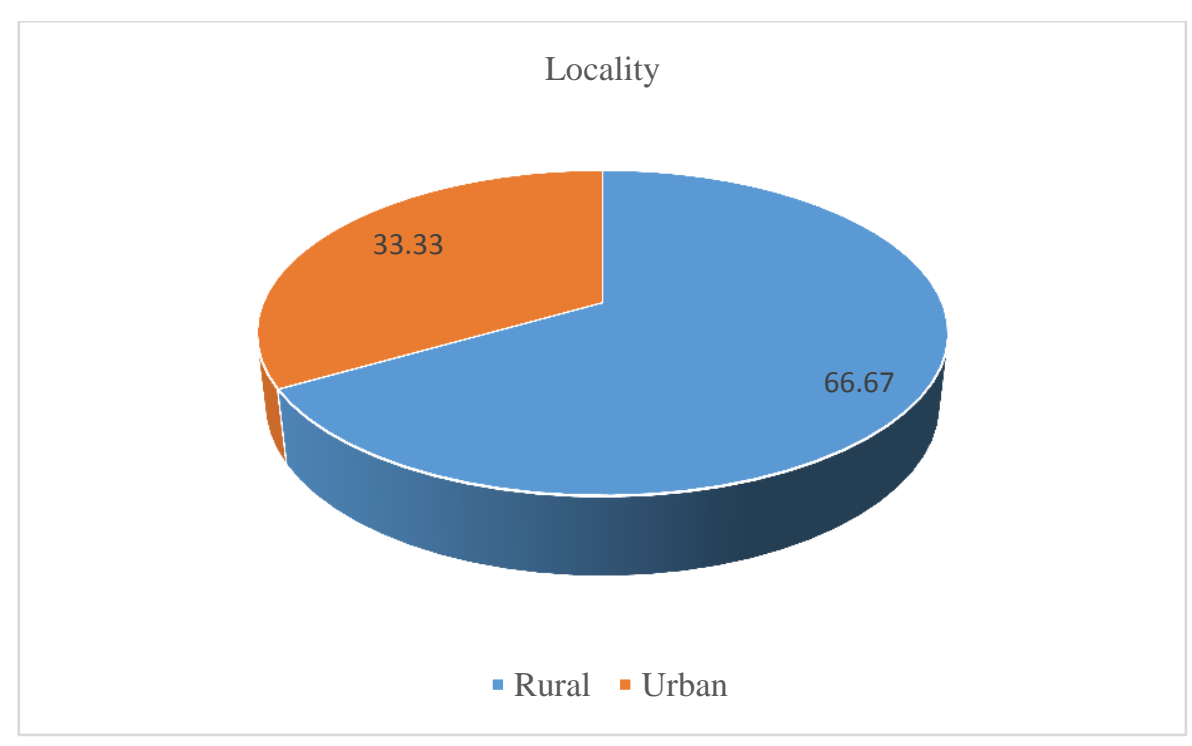

Figure: 1.4 
Table 2: MMSE score distribution among subjects $\mathrm{N}=\mathbf{5 4}$

\begin{tabular}{|l|l|l|l|l|}
\hline Mini Mental & Distribution & Frequency & Percent & Mean MMSE Score+/-SD \\
\cline { 2 - 4 } State Examination (MMSE) & Mild (18-24) & 28 & 51.85 & \multirow{3}{*}{$16.12+/-4.778$} \\
\cline { 2 - 4 } & Moderate(10-17) & 20 & 37.03 \\
\cline { 2 - 4 } & Severe(9 or less) & 6 & 11.12 & \\
\cline { 2 - 4 } & Total & 54 & 100 & \\
\hline
\end{tabular}

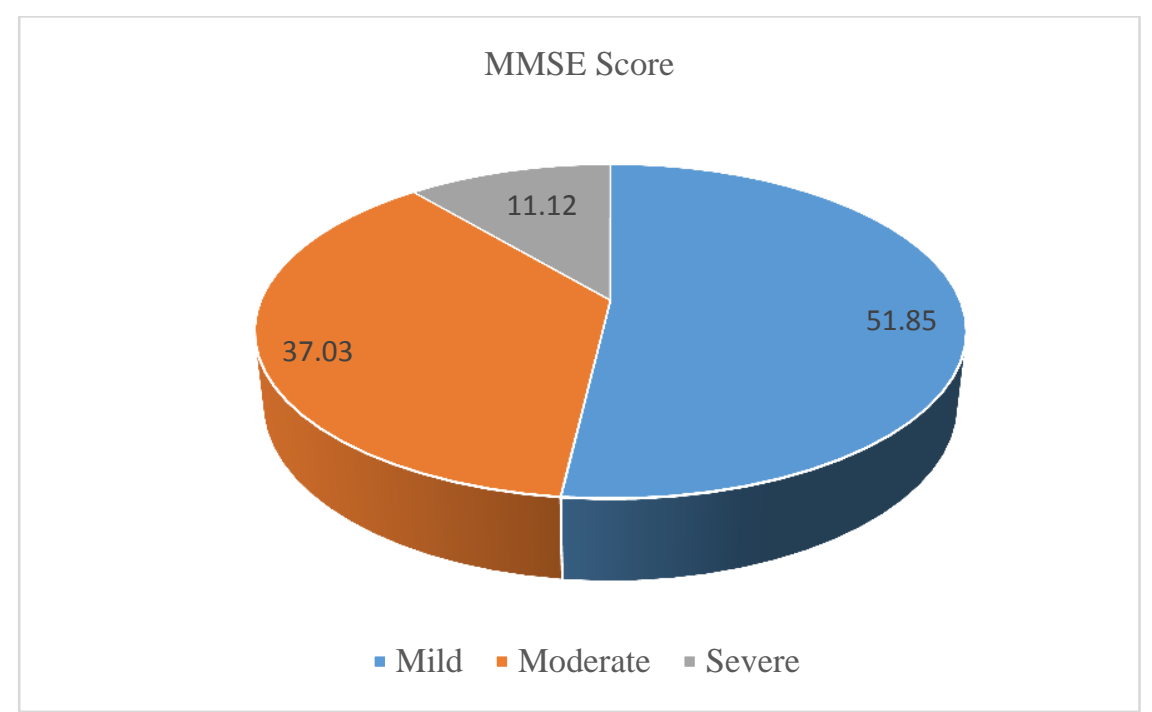

Table 3: Distribution of frequency and mean scores of individual items of NPI

\begin{tabular}{|l|l|l|l|l|}
\hline NPI item & Frequency & Percentage & Mean item score+/-SD & Mean distress score +/-SD \\
\hline Delusions & $\mathrm{N}=14$ & 26 & $1.56+/-2.81$ & $0.94+/-1.70$ \\
\hline Hallucinations & $\mathrm{N}=7$ & 13 & $0.70+/-1.90$ & $0.45+/-1.2$ \\
\hline Agitation & $\mathrm{N}=35$ & 64.8 & $3.40+/-3.05$ & $2.14+/-1.85$ \\
\hline Dysphoria & $\mathrm{N}=30$ & 55.56 & $2.63+/-2.83$ & $1.72+/-1.70$ \\
\hline Anxiety & $\mathrm{N}=30$ & 55.56 & $2.87+/-2.98$ & $1.94+/-1.95$ \\
\hline Elation & $\mathrm{N}=6$ & 11.12 & $0.56+/-1.70$ & $0.40+/-1.23$ \\
\hline Apathy & $\mathrm{N}=38$ & 70.37 & $3.94+/-3.36$ & $2.46+/-1.89$ \\
\hline Disinhibition & $\mathrm{N}=7$ & 13 & $0.56+/-1.51$ & $0.40+/-1.07$ \\
\hline Irritability & $\mathrm{N}=31$ & 57.4 & $3.12+/-3.06$ & $2.01+/-1.90$ \\
\hline Aberrant Motor Behavior & $\mathrm{N}=25$ & 46.3 & $2.14+/-2.61$ & $1.35+/-1.62$ \\
\hline Sleep & $\mathrm{N}=32$ & 59.25 & $3.25+/-3.21$ & $2.09+/-1.96$ \\
\hline Appetite & $\mathrm{N}=30$ & 55.56 & $2.90+/-2.97$ & $1.78+/-1.73$ \\
\hline Total scores & & & $27.84+/-17.58$ & $17.72+/-8.99$ \\
\hline
\end{tabular}

\section{Discussion}

In our present study, $53.70 \%$ are females and $46.30 \%$ are males (Figure 1.1). 61.12\% were above 65 years of age and $38.88 \%$ were under 65 years of age (Figure 1.2). Most of them $51.85 \%$ were illiterate (Figure 1.3). 66.67\% belong to rural background as shown in figure 1.4. 59.25\% came from nuclear families while 40.75 reside in joint families. Mean age of presentation of dementia was $70.92+/-9.44$ years. In our study, $81.48 \%$ patients were having income above 30,000rs. Findings were similar to a study conducted by Jelastopulu et al. in which mean age was 73.6 years. ${ }^{[12]}$

A study was conducted by Patel and Singh in 2012-13 in which males with cognitive impairment were $44.8 \%$ and females were $55.2 \%$. Most of patients in this study, $(71.1 \%)$ belong to rural background. Most of patients $(50.4 \%)$ were illiterate. ${ }^{[13]}$

Majority of patients (92.6\%) in our study did not have any past psychiatric history. Almeida et al reported no association between past depression and incidental cognitive impairment. ${ }^{[14]}$

MMSE score distribution showed that $51.85 \%$ belong to mild dementia group, $37.03 \%$ belong to moderate dementia group and $11.12 \%$ belong to severe dementia group (Figure 2). Mean MMSE score was $16.12+/-4.778$ SD (Table-2) which comes in moderate dementia group.

On Neuropsychiatric Interview (NPI) in our study, 70. 37\% showed apathy, 64.8\% showed agitation, 59.25\% showed sleep disturbances, $57.4 \%$ showed irritable behavior, $55.56 \%$ showed dysphoric symptoms, anxiety symptoms and appetite and eating abnormalities. Less common symptoms were aberrant motor behavior in $46.3 \%$, delusions $26 \%$, hallucinations and disinhibition in $13 \%$ and elation in $11.12 \%$. Mean item score was $27.84+/-17.58$ SD. Mean distress score was 17.72+/-8.99 SD.

In a study conducted by Adreesh Mukherjee et al. similar findings were present. Of a total 107 patients, nearly all (99.1\%) had at least one BPSD. The overall NPI 12-item score was $27.42 \pm$ 18.24 (range 0-72). Of the individual BPSD domains, apathy/indifference was the most frequent $(72.9 \%)$, followed by agitation/ aggression (68.2\%), irritability/lability (59.8\%), sleep and nighttime behaviour disorders $(58.9 \%)$, depression/dysphoria $(56.1 \%)$, appetite and eating disorders (52.3\%), and anxiety $(51.4 \%){ }^{[15]}$

Limitations of study include small sample size and a larger sample is required to accurately assess the BPSD. Majority of the 
patients were illiterate and that might have affected their performance on MMSE. It was a cross sectional study and lacks follow up study of the patients. No control group was taken for comparison.

\section{Conclusion}

Most of patients present with mild to moderate dementia. Behaviour and psychological symptoms were universally present and cause significant caregiver distress.

\section{Data Availability}

The data that support the findings of this study are available from corresponding author [N. B.] upon reasonable request.

\section{Conflicts of Interest}

There is no conflict of interest regarding the publication of this paper.

\section{Funding Statement}

No funding sources

\section{Acknowledgments}

The authors would like to thank the caregivers and persons with dementia who participated in the study.

\section{Bibliography}

1. World Health Organisation. Organic, including symptomatic, mental disorders. In International Classification of Diseases (ICD-10) 10th edition. Geneva: World Health Organization; 2009.

2. Das SK, Pal S, Ghosal MK. Dementia: Indian scenario. Neurology India. 2012;60(6):618-24.

3. Dawood S. Caregiver burden, quality of life and vulnerability towards psychopathology in caregivers of patients with dementia/Alzheimer's disease. Journal of the College Physicians Surgeons Pakistan. 2016;26(11):892-5.

4. Prince M, Guerchet M, Prina M. The global impact of dementia 2013-2050. Alzheimer's Disease International; 2013.
5. World Alzheimer Reports. Alzheimer's Disease International [Internet]. Alz.co.uk. 2015 [cited 6 July 2019]. Available https://www.alz.co.uk/research/WorldAlzheimerReport2 015.pdf2015

6. Finkel SI, e Silva JC, Cohen G, Miller S, Sartorius N. Behavioral and psychological signs and symptoms of dementia: a consensus statement on current knowledge and implications for research and treatment. International Psychogeriatrics. 1997;8(S3):497-500.

7. Cerejeira J, Lagarto L, Mukaetova-Ladinska E. Behavioral and psychological symptoms of dementia. Frontiers in neurology. 2012;3:73.

8. Huang SS, Wang WF, Liao YC. Severity and prevalence of behavioral and psychological symptoms among patients of different dementia stages in Taiwan. Archives of Clinical Psychiatry (São Paulo). 2017;44(4):89-93.

9. Romero AP, Garrido SG. The importance of behavioural and pyschological symptoms in Alzheimer disease. Neurología (English Edition). 2018;33(6):378-84.

10. Sheehan B. Assessment scales in dementia. Therapeutic advances in neurological disorders. 2012;5(6):349-58.

11. Cummings JL. The Neuropsychiatric Inventory: assessing psychopathology in dementia patients. Neurology. 1997;48(5 Suppl 6):10S-6S.

12. Jelastopulu E, Giourou E, Argyropoulos K, Kariori E, Moratis E, Mestousi A, et al. Demographic and clinical characteristics of patients with dementia in Greece. Advances in Psychiatry. 2014;2014:1-7.

13. Patel RM, Singh US. Prevalence study of cognitive impairment and its associated sociodemographic variables using mini-mental status examination among elderly population residing in field practice areas of a medical college. Indian journal of community medicine: official publication of Indian Association of Preventive \& Social Medicine. 2018;43(2):113.

14. Almeida OP, Hankey GJ, Yeap BB, Golledge J, Flicker L. Depression as a risk factor for cognitive impairment in later life: the Health In Men cohort study. International journal of geriatric psychiatry. 2016;31(4):412-20.

15. Mukherjee A, Biswas A, Roy A, Biswas S, Gangopadhyay G, Das SK. Behavioural and psychological symptoms of dementia: correlates and impact on caregiver distress. Dementia and geriatric cognitive disorders extra. 2017;7(3):354-65. 